\title{
EDITORIAL
}

\section{Quantitation of mitral regurgitation: rationale, approach, and interpretation in clinical practice}

\author{
M Enriquez-Sarano, C Tribouilloy
}

Heart 2002;88(Suppl IV):iv1-iv4

Quantitation of mitral regurgitation has made considerable progress is recent years, providing unique insights into the physiology of the condition, and providing essential tools for a proactive modern management of patients with MR

M itral regurgitation (MR) is a valvar haemodynamic alteration which is the focus of intense interest despite the decline of rheumatic disease, because of its high frequency. ${ }^{1}$ Indeed, in our current aging population, MR is most often caused by diseases prevalent in the elderly, either degenerative lesions (such as mitral valve prolapse or ruptured chordae) or functional alterations (the consequence of ventricular dysfunction). ${ }^{2}$ Hence, the assessment of MR is a key task of internists, cardiologists, and cardiac surgeons and has been transformed by Doppler echocardiography. In the past decade new methods and new concepts have been developed for the quantitation of MR, which have been detailed by Irvine and colleagues in this supplement to Heart. ${ }^{3}$ As one may ponder the incremental benefit of new approaches, it is important to review their rationale, to delineate how to integrate them in a global approach, and how to interpret these new results.

\section{RATIONALE FOR THE QUANTITATION OF MR IN CLINICAL PRACTICE} Rationale 1: MR impacts seriously on outcome

Severe MR has the reputation of being well tolerated for many years. Although this is occasionally true, this dogma has little support from outcome data. Indeed, when we examined the long term outcome of patients with flail leaflets that epitomise severe MR, ${ }^{4}$ excess mortality, ${ }^{5}$ and high morbidity ${ }^{6}$ were observed, with high rates of heart failure, atrial fibrillation, ${ }^{7}$ and even sudden death. ${ }^{6}$ It is also remarkable that in functional MR, although the disease is not initially valvar in nature, the higher the degree of MR, the worse the outcome. ${ }^{8}{ }^{9}$ Thus defining the degree of MR is an essential step in the clinical decision making process.

See end of article for authors' affiliations

Dr Maurice

Enriquez-Sarano, Mayo Clinic, 200, First Street SW, Rochester, MN, USA; sarano.maurice@mayo.edu term outcomes with survival similar to that in the general population. ${ }^{11}$ Hence, in patients with repairable valves, surgery is often offered at an early stage, before the development of severe symptoms or ventricular dysfunction. This approach to management requires certainty on the severity of MR.

\section{Rationale 3: classical methods of} assessment of MR have major limitations

Clinical examination is key to management of valve diseases and we observed that high murmur intensity ${ }^{12}$ or a third heart sound ${ }^{13}$ suggest severe MR. However, we have to recognise that the degree of confidence in these observations is imperfect. Although colour flow imaging is sensitive and absent or small jets are reassuring, this technique is misleading with thin eccentric jets often underestimating severe MR and central jets often overestimating moderate MR. ${ }^{14}$ Pulmonary venous flow systolic reversal is specific but is also insensitive as it is dependent on "V wave" formation often absent in chronic MR. ${ }^{15}$ Hence, in patients with more than mild MR, we are often left uncertain and have to resort to fuzzy terminology and to multiplication of tests.

Rationale 4: MR is a continuum best quantified than categorised

No physiologic variable is absolutely fixed and MR may vary in its degree, just like the blood pressure does. Is it sufficient to say "severe MR" like we say "hypertension" or are the numbers important in describing the patient's status? MR is a continuum and two patients with regurgitant volumes of 60 and $100 \mathrm{ml}$, although both with severe MR, are different and may need different therapeutic approaches. In patients with ischaemic MR, the larger the effective regurgitant orifice (ERO) the worse the survival is, ${ }^{8}$ so that clinical decision may be strongly influenced by quantitative measurements.

Most importantly a major rationale for quantifying MR is that this approach is highly feasible in clinical practice.

\section{APPROACH TO QUANTITATION OF MR Approach 1: forming a "Gestalt" on the degree of $M R$ is the first step}

A comprehensive Doppler echocardiography is always in order in patients with MR as discussed by Irvine and colleagues. ${ }^{3}$ A perception on the degree of $\mathrm{MR}$ is formed by delineating the anatomical lesions (a flail leaflet is highly suggestive of severe MR), by observing the left atrial penetration and direction of the jet (an eccentric jet is suspicious for severe MR), by noting the size 


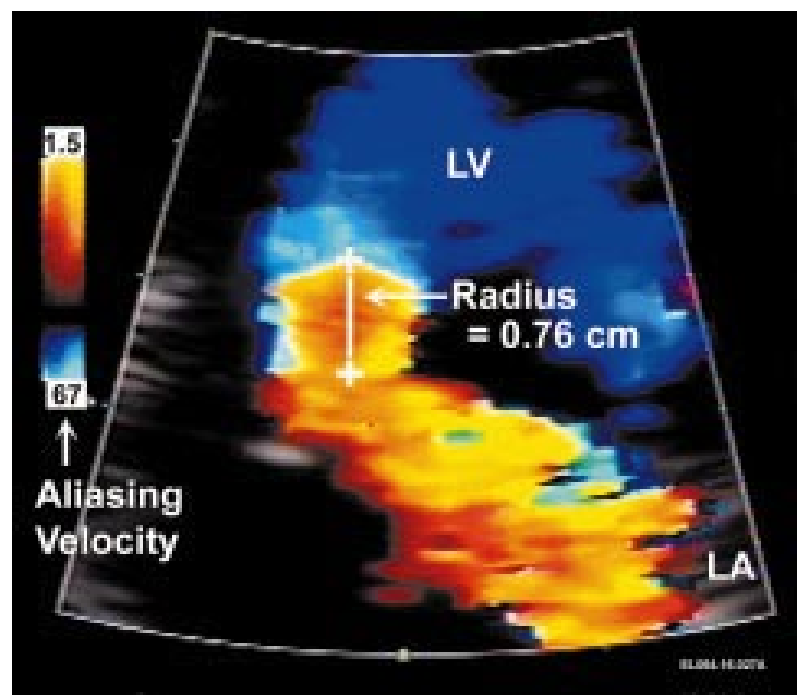

Figure 1 Colour flow imaging of the flow convergence in a patient with a flail posterior leaflet. The baseline of colour has been shifted down and the aliasing velocity (where the blue flow turns to yellow) is indicated by the arrow to the left. The radius of the flow convergence is measured between the crosses. LV, left ventricle; LA, left atrium.

and function of the left ventricle and atrium, and by recording the pulmonary venous flow, mitral inflow and measuring pulmonary pressure.

\section{Approach 2: the "vena contracta" is a simple and useful tool}

The vena contracta is the narrowest neck of the regurgitant flow through and immediately below the regurgitant orifice. ${ }^{16}$ The width of the vena contracta seen by colour flow imaging is a surrogate for the regurgitant orifice size. By positioning the beam of ultrasound perpendicular to the vena contracta, one uses its axial resolution and the method is both simple and useful.

Approach 3: quantifying MR provides physiologic data The preferred method for quantifying MR is the PISA (flow convergence) method. It is simple, fast, reproducible, and has been proven reliable by multiple investigators. ${ }^{17}{ }^{18}$ If it looks good (fig 1) - that is, if the flow convergence appears as a ball sitting on the jet-and if it is properly timed-that is, if it is simultaneous to the peak MR velocity-the results are reliable. ${ }^{17}$ Complexities such as flow convergence constraint and its correction apply to a minority of cases. In our institution approximately $70 \%$ of patients with $M R \geqslant$ moderate, the regurgitation is quantified by this method. Quantitative Doppler and quantitative two dimensional echocardiography with contrast injection are supportive methods that can be averaged with the PISA to ensure narrower range of error. These methods all provide the values of the ERO and regurgitant volume.

Thus, quantitation of MR is rationale and feasible but how are we going to use it?

\section{UTILISATION AND INTERPRETATION OF QUANTITATIVE MR DATA}

Although we may wish to use these tests in all patients with $\mathrm{MR}$, we need to carefully analyse how to best use quantitation of MR.

\section{When is it necessary?}

It is not necessary to quantify MR when the jet is extremely small $\left(<4 \mathrm{~cm}^{2}\right)$ as in such patients MR is small enough to have little or no impact on management. When the MR appears severe, the value of quantitation may be disputed but it is of particular importance with minimal symptoms or when the clinical or echocardiographic data are discordant (such as with mild or absent ventricular or atrial dilatation). Moderate MR covers a wide range and quantitation is helpful in defining patients with the largest degree of MR who will need the closest follow up. Hence, in our practice quantitation of MR is often necessary.

\section{What do these numbers mean?}

Interpretation of quantitative data has common principles but also needs to be performed in the specific patient context. First, it should be understood that the ERO measures the lesion severity, while the regurgitant volume measures the volume overload. This concept is particularly important in acute MR, because the non-compliant cavities limit the volume overload and the regurgitant energy is transformed into potential energy (pressure) rather than into kinetic energy (regurgitant volume). Both measures are therefore important to understand and manage the situation. Second, what we call severe organic MR is defined by a regurgitant volume $\geqslant 60 \mathrm{ml} /$ beat and an ERO $\geqslant 40 \mathrm{~mm}^{2}$ or $0.40 \mathrm{~cm}^{2}$. ${ }^{19}$ However, in functional and ischaemic MR lower thresholds are associated with poor outcome. Indeed patients with ERO $\geqslant 20 \mathrm{~mm}^{2}$ or $0.20 \mathrm{~cm}^{2}$ display considerable excess mortality, so that such a threshold should be considered as the marker for severe ischaemic MR. Therefore, the numbers obtained by quantitation of MR allow us to have a common language and allow us to achieve the most important goal of our profession-that is, to define the severity of the disease one patient at a time and to individualise the management according to these data.

In summary, quantitation of MR has made considerable progress, is highly feasible and reliable, gives unique insights into MR physiology, and provides essential tools for a proactive modern management of patients with MR.

\section{Authors' affiliations}

M Enriquez-Sarano, Mayo Clinic, Rochester, Minnesota, USA

C Tribouilloy, Centre Hospitalo-Universitaire, Amiens, France

\section{REFERENCES}

1 Singh J, Evans J, Levy D, et al. Prevalence and clinical determinants of mitral, tricuspid and aortic regurgitation. Am J Cardiol 1999:83:897-902.

2 Olson L, Subramanian R, Ackermann D, et al. Surgical pathology of the mitral valve: a study of 712 cases spanning 21 years. Mayo Clin Proc 1987;62:22-34.

3 Irvine T, Li XK, Sahn DJ, et al. Assessment of mitral regurgitation. Heart 2002;88(suppl IV):iv1 1-19.

4 Himelman R, Kusumoto F, Oken K, et al. The flail mitral valve: echocardiographic findings by precordial and transesophageal imaging and Doppler color flow mapping. J Am Coll Cardiol 1991;17:272-9.

5 Ling H, Enriquez-Sarano M, Seward J, et al. Clinical outcome of mitral regurgitation due to flail leaflets. N Eng J Med 1996;335:1417-23.

6 Grigioni F, Enriquez-Sarano M, Ling L, et al. Sudden death in mitral regurgitation due to flail leaflet. J Am Coll Cardiol 1999;34:2078-85.

7 Grigioni F, Avierinos JF, Ling LH, et al. Atrial fibrillation complicating the course of degenerative mitral regurgitation. Determinants and long-term outcome. J Am Coll Cardiol 2002;40:84-92.

8 Grigioni $\mathbf{F}$, Enriquez-Sarano M, Zehr K, et al. Ischemic mitral regurgitation: long-term outcome and prognostic implications with quantitative Doppler assessment. Circulation 2001;103:1759-64

9 Hickey M, Smith L, Muhlbaier L, et al. Current prognosis of ischemic mitral regurgitation. Implications for future management. Circulation 1988;78-1:51-9.

10 Carpentier A. Cardiac valve surgery - the "French Correction". J Thorac Cardiovasc Surg 1983;86:323-37.

11 Enriquez-Sarano $M$, Schaff H, Orszulak T, et al. Valve repair improves the outcome of surgery for mitral regurgitation. Circulation 1995:91:1264-5.

12 Desjardins V, Enriquez-Sarano M, Tajik A, et al. Intensity of murmurs correlates with severity of valvular regurgitation. Am J Med 1996; 100:149-56.

13 Tribouilloy CM, Enriquez-Sarano M, Mohty D, et al. Pathophysiologic determinants of third heart sounds: a prospective clinical and Doppler echocardiographic study. Am J Med 2001;111:96-102. 
14 Chen C, Thomas J, Anconina J, et al. Impact of impinging wall jet on color Doppler quantification of mitral regurgitation. Circulation 1991;84:712-20.

15 Enriquez-Sarano M, Dujardin K, Tribouilloy C, et al. Determinants of pulmonary venous flow reversal in mitral regurgitation and its usefulness in determining the severity of the mitral regurgitation. Am J Cardiol 1999:83:535-41.

16 Tribouilloy C, Shen W, Quere J, et al. Assessment of severity of mitral regurgitation by measuring regurgitant jet width at its origin with transesophageal Doppler color flow imaging. Circulation $1992: 85: 1248-53$
17 Enriquez-Sarano M, Miller FJ, Hayes S, et al. Effective mitral regurgitant orifice area: clinical use and pitfalls of the proximal isovelocity surface area method. J Am Coll Cardiol 1995;25:703-9.

18 Vandervoort $\mathbf{P}$, Rivera J, Mele D, et al. Application of color Doppler flow mapping to calculate effective regurgitant orifice area. An in vitro study and initial clinical observations. Circulation 1993;88:1150-6.

19 Dujardin K, Enriquez-Sarano M, Bailey K, et al. Grading of mitral regurgitation by quantitative Doppler echocardiography - calibration by left ventricular angiography in routine clinical practice. Circulation 1997:96:3409-15

\section{IMAGES IN ECHOCARDIOGRAPHY.}

\section{Perforated mitral valve leaflet}

A

40 year old man presented with general malaise, pyrexia, and microembolic phenomena. Blood cultures were positive for $\alpha$ haemolytic streptococcus. A transthoracic echocardiogram showed several small masses on the aortic valve (AV), consistent with vegetations, and severe aortic regurgitation (AR). A large mass was seen on the atrial aspect of the anterior mitral valve leaflet (AMVL) thought to be a large vegetation. A transoesophageal echocardiogram was performed confirming AV vegetations with severe AR and an AMVL aneurysm with associated leaflet perforation and severe mitral regurgitation. The site of AMVL perforation corresponded to the point of impact of the AR jet on the leaflet, creating a "jet lesion". The primary lesion was therefore AV endocarditis with secondary involvement of the mitral valve. The patient's haemodynamic state deteriorated and he subsequently underwent aortic and mitral valve replacement.

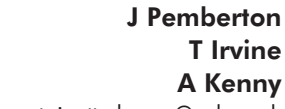

antoinette.kenny@ncl.ac.uk

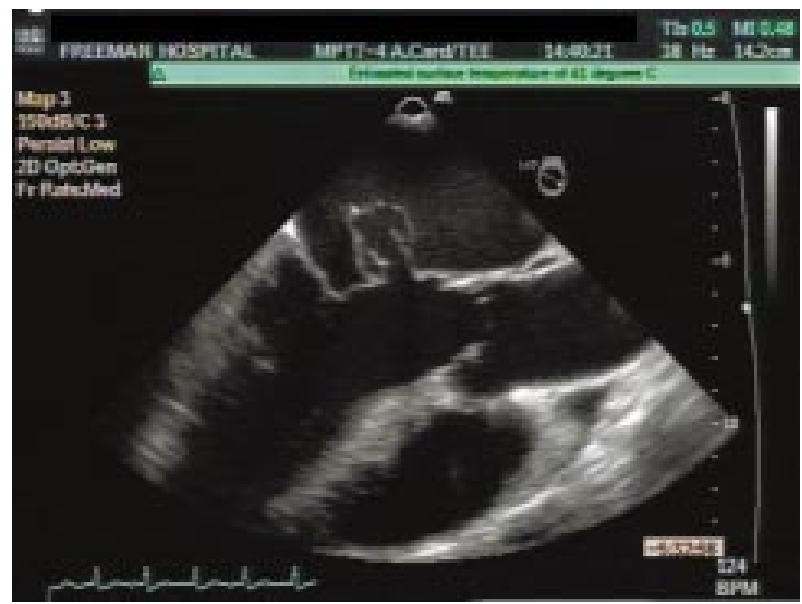

Figure 1 Perforation in the anterior mitral valve leaflet (AMVL)

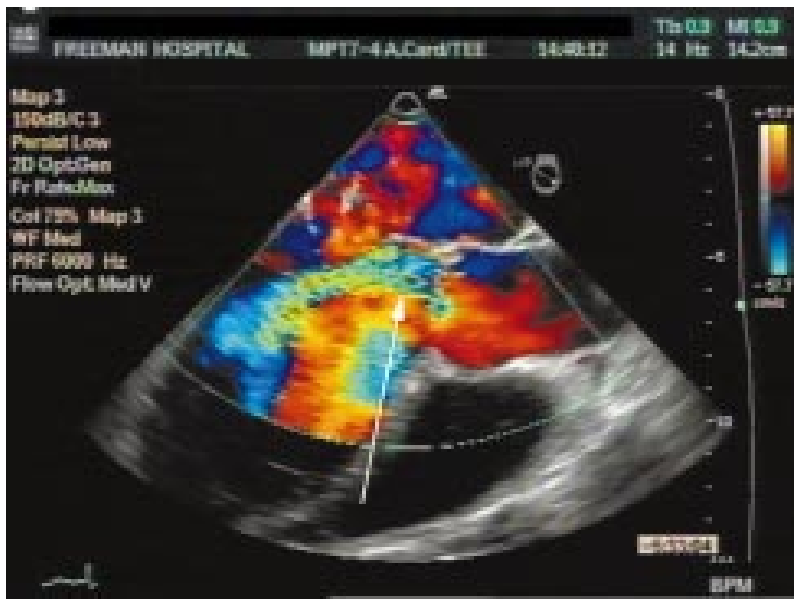

Figure 2 Jet of aortic regurgitation (arrow) directed against the AMVL.

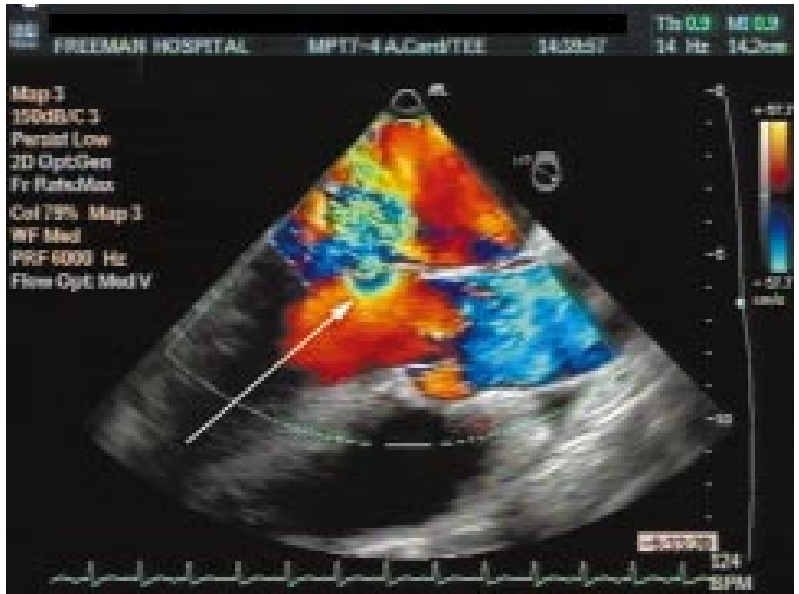

Figure 3 Severe mitral regurgitation (arrow) through the perforated leaflet. 


\section{IMAGES IN ECHOCARDIOGRAPHY.}

\section{Stuck mitral valve replacement}

T

his 30 year old woman had undergone a mitral valve replacement with a bileaflet prosthesis one year previously. Postoperatively she had been well until she presented with a two week history of shortness of breath and an episode of paroxysmal nocturnal dyspnoea. During this time her anticoagulation had been subtherapeutic. A transoesophageal echocardiogram (TOE) was performed to assess the prosthetic mitral valve. The TOE showed a stuck leaflet caused by thrombus with flow through only one side of the prosthesis.
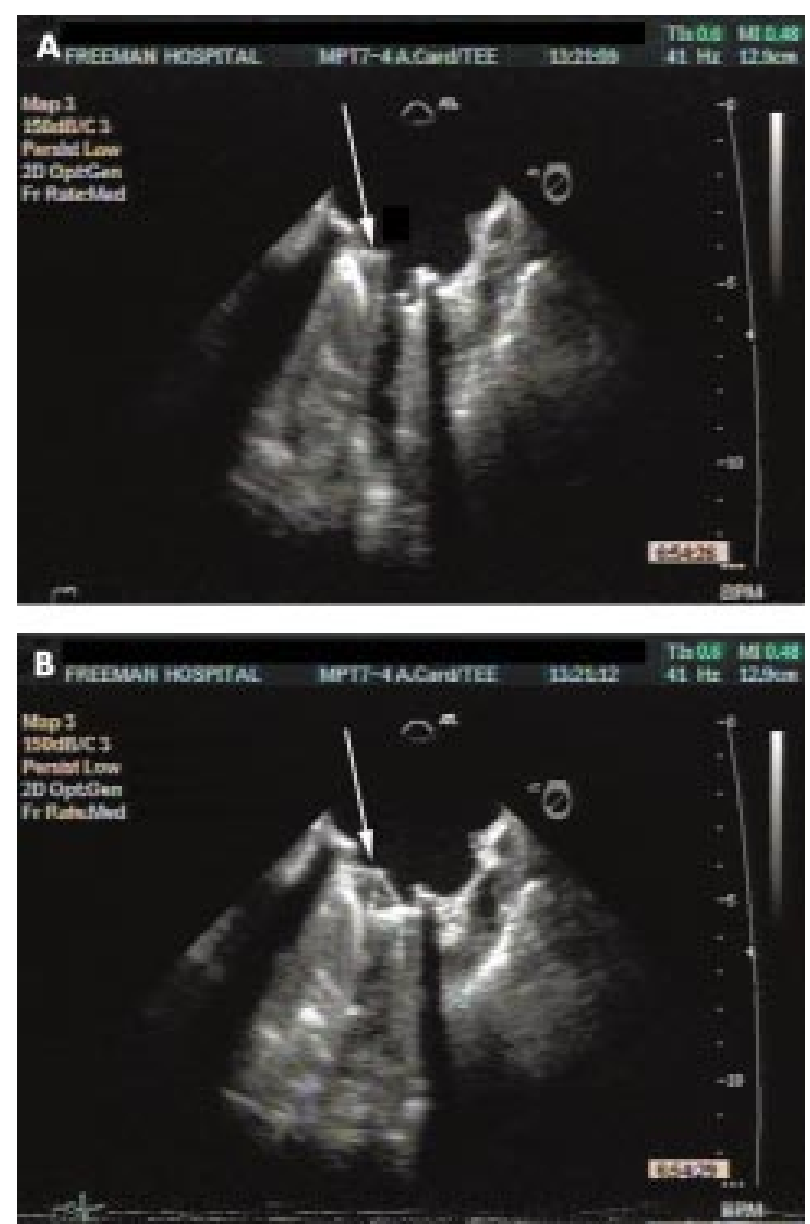

Figure 1 Systolic and diastolic frames showing open and stuck (arrow) mitral valve leaflets.
Continuous wave Doppler through the valve gave an elevated peak forward flow velocity of $3 \mathrm{~m} / \mathrm{s}$. After a period of adequate anticoagulation the valve was unchanged and the patient underwent redo mitral valve replacement.

J Pemberton

T Irvine

A Kenny

antoinette.kenny@ncl.ac.uk

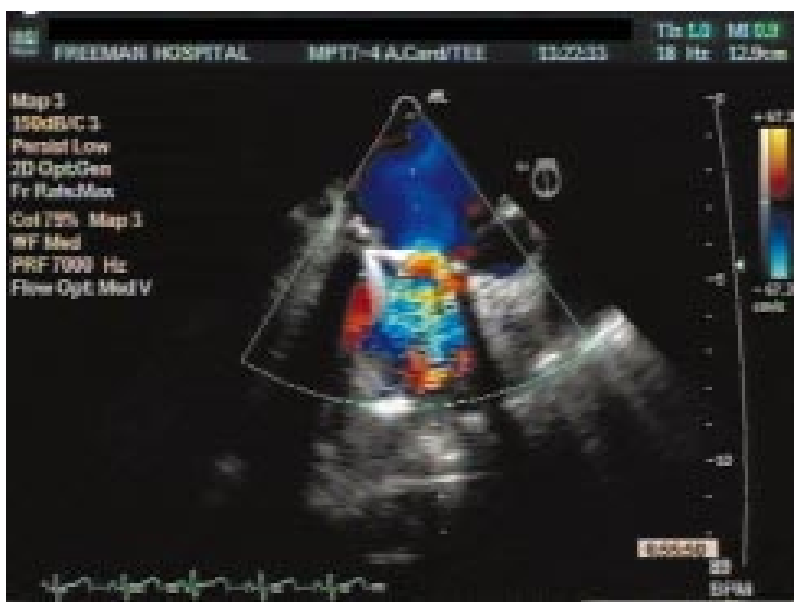

Figure 2 Asymmetric colour flow through single open mitral valve leaflet.

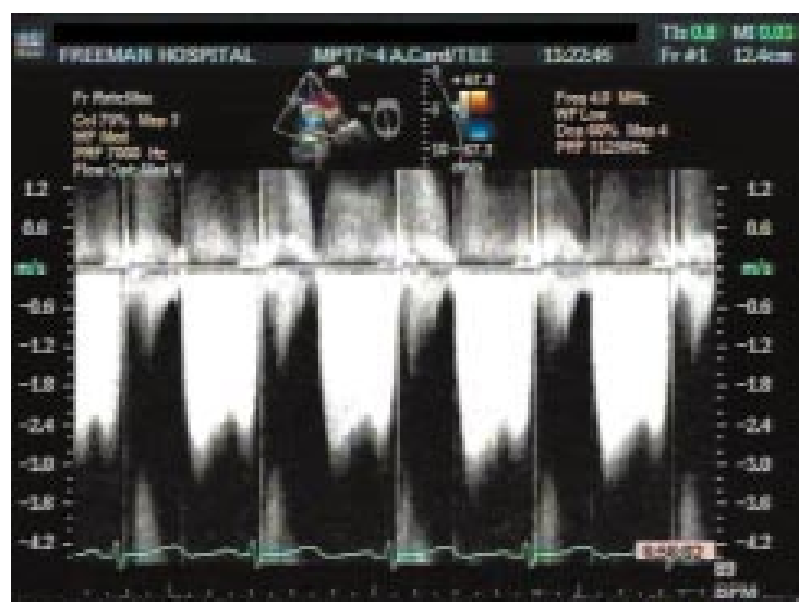

Figure 3 Continuous wave Doppler through mitral valve with peak forward flow velocity of $3 \mathrm{~m} / \mathrm{s}$. 